\section{Military Technical College Kobry El-Kobbah, Cairo, Egypt}

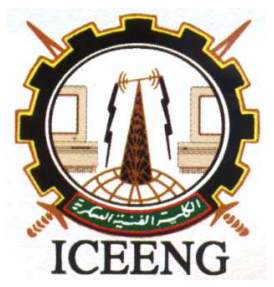

\author{
$6^{\text {th }}$ International Conference \\ on Electrical Engineering \\ ICEENG 2008
}

\title{
Application of supercapacitor technologies in distribution systems
}

$$
\text { By }
$$

\author{
W. Sabry*
}

S. Gawish **

\section{Abstract:}

Supercapacitors are neutral systems that contain an electric double layer at the interface of the metal electrode and electrolyte dielectric material. The neutrality of the system is obtained by a diffuse layer of positive charges in the electrolyte opposing a centralized layer of negative charges in the electrode. Thus, the double layer is formed in which the separation of each layer is on the order of a few angstroms. As a first trial, in this paper the technology of supercapacitors is applied theoretically for the electrical power distribution systems. A simple distribution system (two-node system) is proposed. The system represents the one line diagram of a source - line - load system. By increasing the capacitance of the supercapacitor at the load bus, the load flow analysis results showed that the over voltage problem and also the high losses problem at load are removed.

\section{Keywords:}

Electrolyte, super capacitor, distribution system 


\section{Introduction:}

Capacitors are designed to provide energy in an electric circuit by storing energy in an electric field that is between two separate conducting plates or electrodes. When applying a voltage source to a capacitor, electrons are forced onto the surface of one electrode that in turn pulls electrons off the surface of the other.

The used dielectric material depends on the application and includes paper, ceramic, air, mica, and electrolytic materials. Because the dielectric material between the plates act as an electron insulator, electrical current does not flow until an external circuit is closed resulting in a discharge.

Electrolyte dielectric materials serve both as an insulator to prevent current flow and as a media to support the electrostatic force of a charged capacitor electrode. Dielectrics are rated by their ability to support the electrostatic forces by employing a number termed dielectric constant.

The standard by which all other dielectric materials are measured is a vacuum, which has a dielectric constant of 1.0 The value for air is approximately the same which is equal to 1.001 and for water is 80.4. However, a variety of materials can be used as dielectric materials with different dielectric constants [1].

Factors that affect the value of capacitance include the area of the plates, the distance between the plates and the dielectric constant of the material between the plates. When the plates have a larger surface area, they provide a greater capacity to store a charge, thus a higher capacitance. The electrostatic force field between the plates is much stronger when they are closer together and the value of capacitance is directly proportional to the amount of electrostatic force. Therefore, as the distance between the plates increases, the capacitance decreases.

The third factor determining capacitance is the dielectric material. There is a direct correlation between the ability of the dielectric material that supports the electrostatic forces to the dielectric constant. Therefore, as the dielectric constant decreases, capacitance decreases.

The capacitance generated in polymeric transducers has been determined to be due to the electric double layer that forms between the interface of the electrode and dielectric polymer. The electric double layer theory has been used to calculate the motion of ions and distribution of ions in an electrolyte dielectric material. The mobile ions in the electrolyte tend to be adsorbed onto the metal layer and also tend to be distributed away 
from the polymer-metal interface in such a fashion that the charge on the metal electrode is balanced. This phenomenon is termed super capacitance and is described as having a very large capacitance per unit weight or volume [2].

Supercapacitors are neutral systems that contain an electric double layer at the interface of the metal electrode and electrolyte dielectric material. The neutrality of the system is obtained by a diffuse layer of positive charges in the electrolyte opposing a centralized layer of negative charges in the electrode. Thus, the double layer is formed in which the separation of each layer is on the order of a few angstroms.

\section{Supercapacitors:}

Supercapacitors also referred to as ultra capacitors or electrochemical double layer capacitors or pseudo capacitors, are different from the conventional electrostatic and electrolytic capacitors because they contain an electrolyte, which enables the electrostatic charge to be stored in the form of ions. They are governed by the same fundamental equations as conventional capacitors, but utilize higher surface area electrodes and thinner dielectrics to achieve greater capacitances.

Since these devices store energy using ionic capacitance as well as by surface reactions, their classification lies closer to a conventional battery than its conventional capacitor relatives.

There still some confusion in the literature as the two surnames (super- and ultra-) are often used interchangeably and have vague definitions when used. However, for convenience, this type of device will still be referred to as a super capacitor throughout this paper.

A model of an actual capacitor must include an equivalent series resistance (ESR) to account for internal losses. A schematic of an actual capacitor is shown in figure (1). Depending upon the application, the ESR can have a big impact on the voltage fluctuation across the capacitor during charging, as well as the current leakage rate out of the capacitor over extended periods of inactivity.

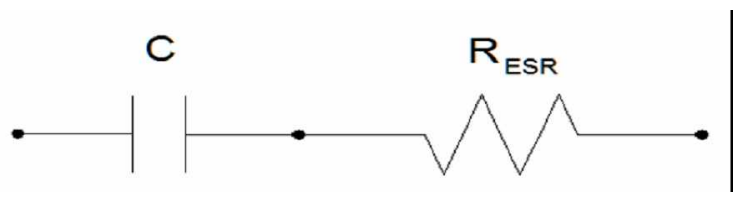

Figure (1): Schematic of realistic capacitor model incorporating ESR 
The most recent development in supercapacitors is employing electrochemical energy storage similar to methods used in batteries. One of the most common types of super capacitor is known as the electrochemical double layer capacitor, or EDLC. Because no transfer of charge between the electrolyte and the electrode occurs, the charge storage in EDLCs is highly reversible, accounting for their stable performance characteristics for as many as 106 cycles. In contrast, the cycle life of electrochemical batteries is generally limited to a maximum of 103 cycles [3].

To demonstrate the impact of ESR on storage properties, two supercapacitors were tested, the first rated at $0.22[\mathrm{~F}]$ and $5.5[\mathrm{~V}]$, with a reported equivalent series resistance of $75[\Omega]$ the second capacitor was rated at $0.3[\mathrm{~F}]$ and $4.5[\mathrm{~V}]$, with an equivalent series resistance of 34 [m $\Omega$ ]. For a discharge experiment for both, the first is completely discharged at 160 second and the second is completely discharged at 100 second [4].

That is, when the equivalent series resistance of a supercapacitor decreases, the rate of discharge increases. Also, as a general rule of thumb, the equivalent series resistance decreases as the capacity of the supercapacitor increases.

For all electrochemical energy storage systems, including batteries as well as capacitors, self-discharge is an intrinsic property, occurring at a higher rate for supercapacitors. Therefore, if ambient energy is only available for a small portion of the day, a super capacitor may have too high a self-discharge rate to be useful for wireless applications. On the other hand, if multiple sources of ambient energy are harvested, supercapacitors might be more desirable for energy storage and electrical power distribution system.

\section{Features and technology of supercapacitors:}

Such supercapacitors have several advantages relative to batteries; very high rates of charge and discharge, little degradation over hundreds of thousands of cycles, good reversibility, low toxicity of materials used and high cycle efficiency (95\% or more).

On the other hand, supercapacitors have several disadvantages; the amount of energy stored per unit weight is considerably lower than that of an electrochemical battery (3-5 $\mathrm{Wh} / \mathrm{kg}$ for an supercapacitor compared to $30-40 \mathrm{Wh} / \mathrm{kg}$ for a battery) which is also only about $1 / 10,000$ th the volumetric energy density of gasoline, the voltage varies with the energy stored to effectively store and recover energy requires sophisticated electronic control and switching equipment and has the highest dielectric absorption of all types of capacitors. 
Figure (2) shows the energy density versus power density for various energy-storage devices. It is clear that supercapacitors has a medium energy density lies in a region between conventional capacitors and conventional batteries; but also has a high power density.

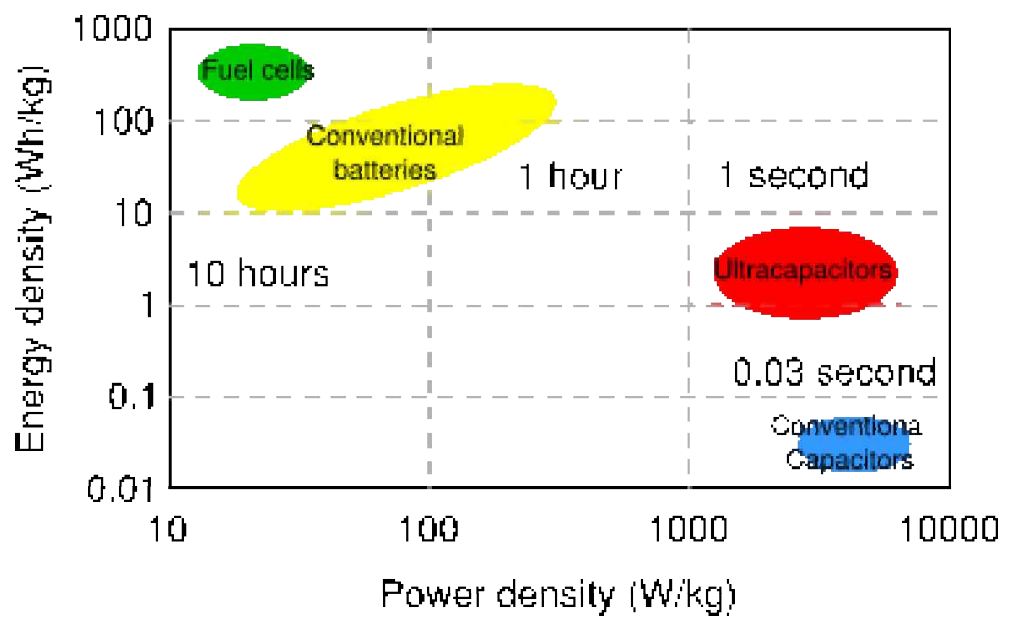

Figure (2): Energy density vs. power density for various energy-storage devices

Figure (3) shows the comparison of construction diagrams of three capacitors. Left: normal capacitor, middle: electrolytic capacitor and right: supercapacitor.

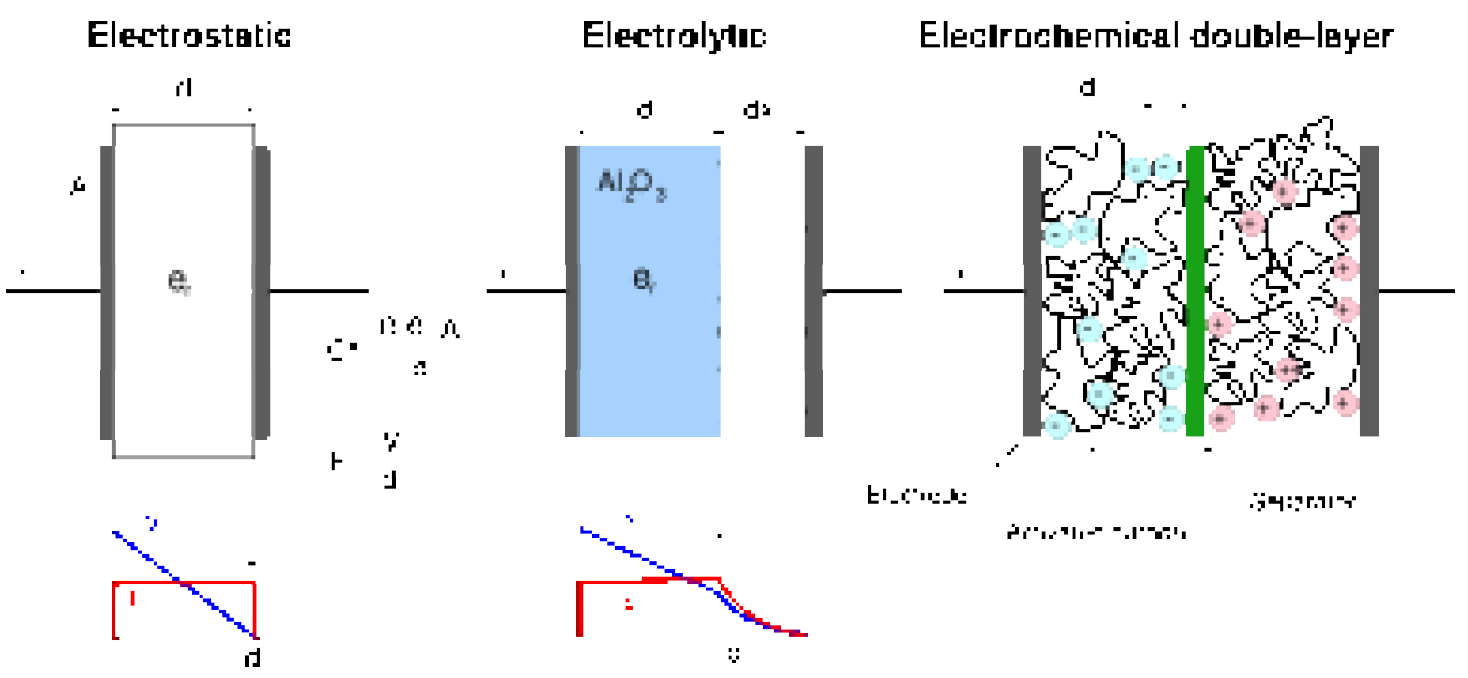

Figure (3): Comparison of construction diagrams of three capacitor types 
Carbon nanotubes and certain conductive polymers, or carbon aerogels, are practical for supercapacitors. Carbon nanotubes have excellent nanoporosity properties, allowing tiny spaces for the polymer to sit in the tube and act as a dielectric. Some polymers like polyacenes, have a redox (reduction-oxidation) storage mechanism along with a high surface area. MIT's Laboratory of Electromagnetic and Electronic Systems (LEES) is researching using carbon nanotubes.

Supercapacitors are also being made of carbon aerogel. Carbon aerogel is a unique material providing extremely high surface area of about $400-1000 \mathrm{~m}^{2} / \mathrm{g}$. Small aerogel supercapacitors are being used as backup batteries in microelectronics, applications for electric vehicles and applications of utility grids are expected.

The electrodes of aerogel supercapacitors are usually made of non-woven paper made from carbon fibers and coated with organic aerogel, which then undergoes pyrolysis. The paper is a composite material where the carbon fibers provide structural integrity and the aerogel provides the required large surface.

The capacitance of a single cell of a supercapacitor can be as high as $3[\mathrm{kF}]$ and some researches tells that this value reaches to $20[\mathrm{kF}]$ [5] .As a first trial, in this thesis and in this chapter, next section, the technology of supercapacitors is applied theoretically for the electrical power distribution systems.

\section{Supercapacitors in distribution systems:}

A simple distribution system (two-node system) is proposed in figure (6.4). The system represents the one line diagram of a source - line - load system. 4. The desired load is $\mathrm{S}$ $=20+\mathrm{j} 5[\mathrm{MVA}]$ which is fed from a source (generator or transformer or substation) with generated power at $11 \angle 0^{\circ} \mathrm{kV}$. The line (feeder) connecting both is of length 2 $[\mathrm{km}]$ and with series resistance of $0.15[\Omega / \mathrm{km}]$ and series reactance of $0.8[\Omega / \mathrm{km}]$. The initial load flow analysis indicates some problems in the line and the unregulated PQbus, bus number (2), which is colored with red. 


\section{Two Nodes Example}

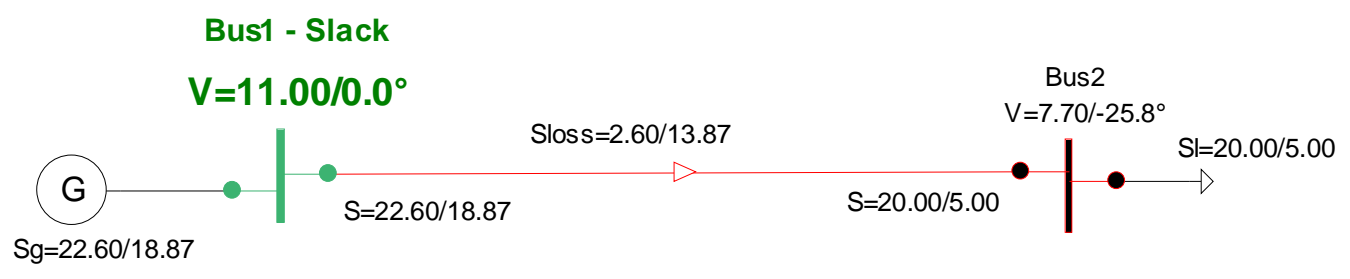

Figure (4): Proposed system under study

Using a supercapacitor of $3[\mathrm{kF}]$ at the load bus which has a reactive power of 12 [MVAR], the load flow analysis results is shown in figure (6.5). The overvoltage problem at load bus is removed but the high losses problem at the line still exists.

\section{Two Nodes Example}

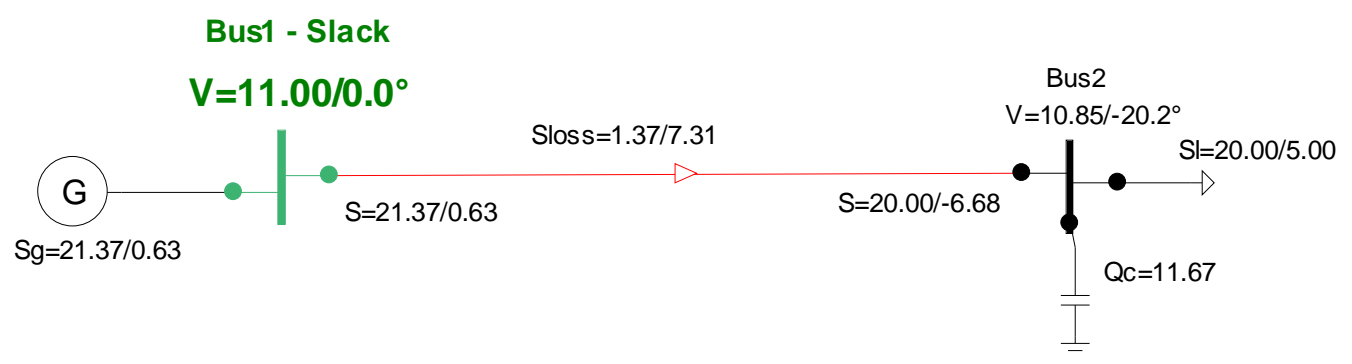

Figure (5): Proposed system after compensation

By increasing the capacitance of the supercapacitor at the load bus to $5[\mathrm{kF}]$, which has a reactive power of about 20 [MVAR], the load flow analysis results is shown in figure (6). The high losses problem at load bus is merely removed. 


\section{Two Nodes Example}

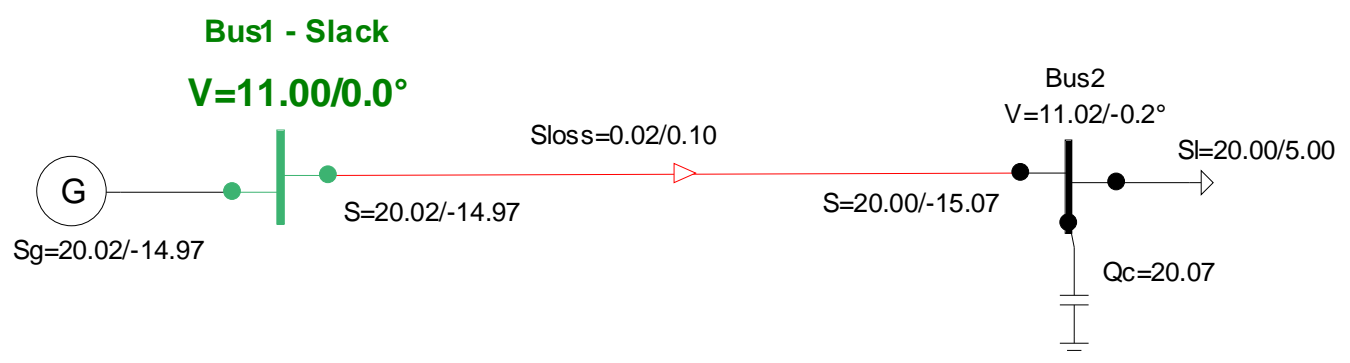

Figure (6): Proposed system after new compensation

By increasing the capacitance of the supercapacitor at the load bus to $6[\mathrm{kF}]$, which has a reactive power of about 24 [MVAR], the load flow analysis results is shown in figure (7). The high losses problem at load bus is now completely removed with ideal load bus voltage.

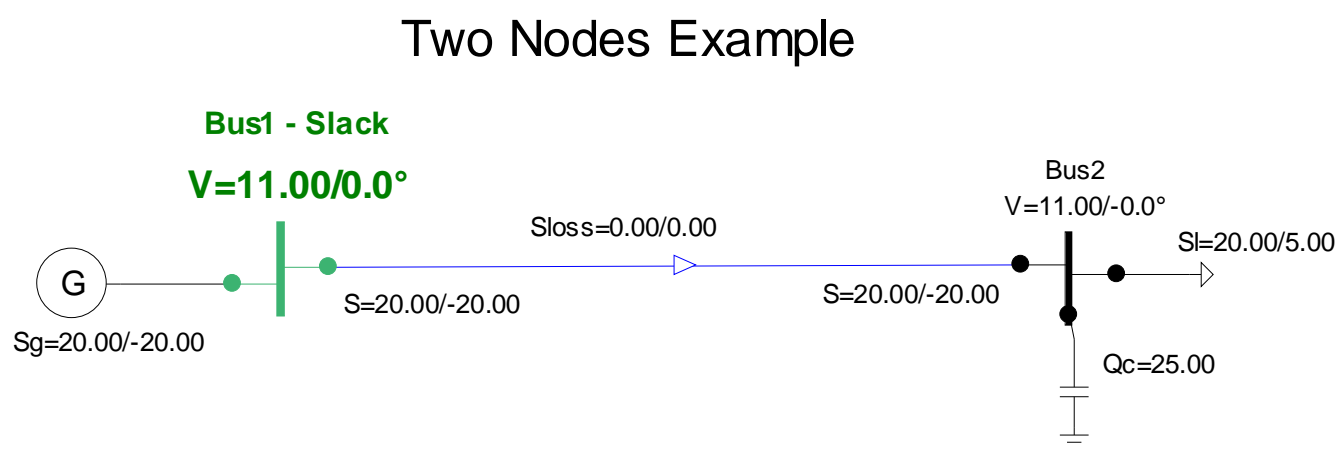

Figure (7): Proposed system after another new compensation

\section{Conclusions:}

In electrical power distribution system, there are many different new trends in manufacturing technology of system components. One of these trends is the supercapacitors technology. In this paper a small trial, for using a supercapacitor in a simple distribution system. This trial was succeeded, because by increasing the capacitance of the supercapacitor at the load bus, the load flow analysis results showed that the over voltage problem and also the high losses problem at load are removed. 


\section{References:}

[1] Sears, F. W., Zemansky, M. W. and Young, H. D. "University Physics", 6 ${ }^{\text {th }}$ Ed., Addison-Wesley, 1982.

[2] Winter, M. and Brodd "What Are Batteries, Fuel Cells and Supercapacitors", R. J. Chem. Rev., 2004.

[3] Halper, M. S. and Ellenbogen, J. C. "Supercapacitors: A Brief Overview", MITRE, McLean, Virginia, 2006.

[4] Justin R. Farmer "A comparison of power harvesting techniques and related energy storage issues", M.Sc. Dissertation submitted to the Faculty of the Virginia Polytechnic Institute and State University, Blacksburg, VA, USA, www.vt.edu, May 2007.

[5] www.mit.edu "MIT Technology Review", Jan, 22, 2007. 